\title{
openheart Atrial fibrillation detected at screening is not a benign condition: outcomes in screen-detected versus clinically detected atrial fibrillation. Results from the Prevention of Renal and Vascular End-stage Disease (PREVEND) study
}

\author{
Victor W Zwartkruis (D) , ${ }^{1}$ Bastiaan Geelhoed, ${ }^{1}$ Navin Suthahar (D) , ${ }^{1}$ \\ Stephan J L Bakker (D) , 2 Ron T Gansevoort, ${ }^{2}$ Isabelle C van Gelder, ${ }^{1}$ \\ Rudolf A de Boer (D) , ${ }^{1}$ Michiel Rienstra (D) ${ }^{1}$
}

- Additional supplemental material is published online only. To view, please visit the journal online (http://dx.doi.org/10. 1136/openhrt-2021-001786).

To cite: Zwartkruis VW, Geelhoed B, Suthahar N, et al. Atrial fibrillation detected at screening is not a benign condition: outcomes in screendetected versus clinically detected atrial fibrillation. Results from the Prevention of Renal and Vascular End-stage Disease (PREVEND) study. Open Heart 2021;8:e001786.

doi:10.1136/

openhrt-2021-001786

Part of the work was presented during the EHRA 2021 Online Congress and published as a conference abstract (EP Europace, Volume 23, Issue Supplement_3, May 2021, euab116.144, https://doi.org/10 1093/europace/euab116.144).

Received 14 July 2021 Accepted 29 November 2021

Check for updates

C) Author(s) (or their employer(s)) 2021. Re-use permitted under CC BY-NC. No commercial re-use. See rights and permissions. Published by BMJ.

For numbered affiliations see end of article.

Correspondence to

Professor Michiel Rienstra; $\mathrm{m}$. rienstra@umcg.n

\section{ABSTRACT}

Aims It is unknown whether screen-detected atrial fibrillation (AF) carries cardiovascular risks similar to clinically detected AF. We aimed to compare clinical outcomes between individuals with screen-detected and clinically detected incident AF.

Methods We studied 8265 participants (age $49 \pm$ 13 years, $50 \%$ women) without prevalent $A F$ from the community-based Prevention of Renal and Vascular End-stage Disease (PREVEND) study. By design of the PREVEND study, $70 \%$ of participants had a urinary albumin concentration $>10 \mathrm{mg} / \mathrm{L}$. Participants underwent 12-lead ECG screening at baseline and every 3 years. AF was considered screen-detected when first diagnosed during a study visit and clinically detected when first diagnosed during a hospital visit. We analysed data from the baseline visit (1997-1998) up to the third follow-up visit (2008). We used Cox regression with screen-detected and clinically detected AF as time-varying covariates to study the association of screen-detected and clinically detected AF with all-cause mortality, incident heart failure (HF) and vascular events.

Results During a follow-up of $9.8 \pm 2.3$ years, 265 participants (3.2\%) developed incident AF, of whom 60 (23\%) had screen-detected AF. The majority of baseline characteristics were comparable between individuals with screen-detected and clinically detected AF. Unadjusted both screen-detected and clinically detected AF were strongly associated with mortality, incident $\mathrm{HF}$, and vascular events. After multivariable adjustment, screendetected and clinically detected AF remained significantly associated with mortality (HR $2.21(95 \% \mathrm{Cl} 1.09$ to 4.47 ) vs 2.95 (2.18 to 4.00 ), $p$ for difference $=0.447$ ) and incident HF (4.90 (2.28 to 10.57) vs 3.98 (2.49 to 6.34 ), p for difference=0.635). After adjustment, screen-detected AF was not significantly associated with vascular events, whereas clinically detected AF was (1.12 (0.46 to 2.71) vs 1.92 (1.21 to 3.06), p for difference $=0.283$ ).

Conclusion Screen-detected incident AF was associated with an increased risk of adverse outcomes, especially

\section{Key questions}

What is already known about this subject?

- Screening for atrial fibrillation (AF) improves early detection. However, it remains unclear whether individuals with screen-detected $A F$ have a similar risk of adverse outcomes compared with patients with clinically detected $\mathrm{AF}$.

What does this study add?

- In a large population-based cohort, screen-detected incident AF was associated with an increased risk of adverse outcomes, especially all-cause mortality and heart failure. There were no significant differences in risk between screen-detected AF and clinically detected AF.

How might this impact on clinical practice?

- Current guidelines make no distinction between screen-detected AF or clinically detected AF, and both are generally treated similarly. Given the findings of this study, this approach seems justifiable until ongoing AF screening trials provide more definitive answers. In addition, this study highlights that AF screening trials should focus not only on the prevention of stroke and mortality but also on the prevention of heart failure.

all-cause mortality and incident HF. The risk of outcomes was not significantly different between screen-detected AF and clinically detected AF.

\section{INTRODUCTION}

Atrial fibrillation (AF) is a major risk factor for mortality, heart failure (HF) and vascular events such as myocardial infarction (MI) and stroke. ${ }^{1-3}$ Combined with an estimated lifetime risk of up to 1 in 3 , and a prevalence 
that is increasing even further due to rising life expectancy and rising prevalence of comorbidities, this makes AF a major healthcare challenge. ${ }^{14}$

Early detection and screening for $\mathrm{AF}$ are considered increasingly important, since effective therapies are available and early treatment of AF may improve outcomes. ${ }^{5-9}$ Various screening strategies have been shown to increase the detection rate of AF. ${ }^{1011}$ Nevertheless, studies have yet to convincingly demonstrate that screening and subsequent treatment of AF can actually improve outcomes and prognosis. ${ }^{12-14}$

Despite the fact that unequivocal evidence from outcome trials is still lacking, European guidelines already recommend $\mathrm{AF}$ screening in individuals $\geq 65$ years. ${ }^{1}$ In addition, current guidelines make no distinction between patients with clinically detected AF and patients with screen-detected AF, and, therefore, recommend similar treatment for both groups. ${ }^{1}$ However, it is unclear whether individuals with screen-detected AF are comparable to individuals with clinically detected AF, and whether they have a similar risk of mortality, HF and vascular events. ${ }^{15}$ Therefore, we aimed to compare clinical characteristics and adverse outcomes between individuals with screen-detected AF and clinically detected AF.

\section{METHODS}

\section{Study population and procedures}

The study was performed with data from the PREVEND (Prevention of Renal and Vascular End-stage Disease) cohort study, which has been described in more detail elsewhere. ${ }^{16}{ }^{17}$ In short, PREVEND is a population-based cohort, which was designed to study if increased urinary albumin excretion is associated with renal and cardiovascular (CV) disease. From 1997 to 1998, 8592 communitydwelling individuals from the city of Groningen, The Netherlands were included in the study. A detailed overview of participant recruitment is provided in online supplemental figure S1. By design, approximately $70 \%$ of participants had a urinary albumin concentration (UAC) $>10 \mathrm{mg} / \mathrm{L}$, while the other $30 \%$ had a UAC $<10 \mathrm{mg} / \mathrm{L}$. At baseline, participants underwent various examinations, including anthropometric measurements, assessment of cardiometabolic risk factors, measurement of blood pressure and ankle brachial index and collection of blood and urine. Follow-up visits were performed every 3 years. A 12-lead ECG was made during the baseline visit and every follow-up visit. For the present study, we excluded participants with previously diagnosed AF based on hospital ECGs made prior to the date of inclusion $(n=37)$, participants with AF on their baseline ECG $(n=42)$ and participants without reliable ECG data $(n=248)$, leaving 8265 participants. The PREVEND study was approved by the Medical Ethical Committee of the University Medical Center Groningen and was conducted in accordance with the Declaration of Helsinki. All participants provided written informed consent.

\section{Ascertainment of AF}

Incident AF was diagnosed if atrial fibrillation or flutter was present on a 12-lead ECG obtained during one of the three PREVEND follow-up visits or during an outpatient visit or hospital admission in either of the two hospitals that together cover the whole city of Groningen. All PREVEND ECGs from the baseline visit up to the third follow-up visit as well as all available hospital ECGs (including those obtained prior to study inclusion) were screened for AF using previously described methods, ${ }^{18}$ which are summarised in the online supplemental notes. If first diagnosed at a PREVEND follow-up visit, AF was considered screen-detected. If first diagnosed during a hospital visit, AF was considered clinically detected. When participants had screen-detected AF, their general practitioner (GP) was informed. Further follow-up and treatment of AF were left at the discretion of the GP.

\section{Clinical outcomes, risk factors and comorbidities}

Information regarding vascular events was derived from the Dutch National Medical Registry, which contains hospital discharge diagnoses from all hospitals in the Netherlands. ${ }^{18} 19$ Discharge diagnoses were coded according to the ninth revision of the International Classification of Diseases (ICD-9). Vascular events included acute MI and ischaemic heart disease (ICD-9 codes 410, 411), percutaneous transluminal angioplasty and bypass grafting of the coronary arteries or peripheral vessels (ICD-9 code 36) as well as haemorrhagic and ischaemic stroke (ICD-9 codes 433,434$)$. A committee of HF experts adjudicated all HF cases according to previously published criteria, ${ }^{17}$ which are summarised in the online supplemental notes. Data on mortality were obtained through the municipal registration and Statistics Netherlands..$^{20}$ Risk factors and comorbidities (hypertension, diabetes, peripheral artery disease, history of MI, history of stroke and prevalent HF) were defined using previously described definitions for PREVEND ${ }^{18}$ which are described in the online supplemental notes.

\section{Follow-up}

The follow-up duration was calculated as the time between the baseline screening visit to the last contact date, death or 31 December 2008 (end of the third PREVEND follow-up visit), whichever came first.

\section{Statistical analyses}

Clinical characteristics at baseline were compared between participants with screen-detected AF and clinically detected AF. For continuous data, characteristics were compared using the independent samples $t$ test for normally distributed data or Mann-Whitney $U$ test for non-normally distributed data. For binary data, Pearson's $\chi^{2}$ test or Fisher's exact test was used depending on sample size.

Incidence rates per 1000 person-years with $95 \%$ CIs were calculated for incident $\mathrm{AF}$ as well as the clinical outcomes of interest (death, incident HF and vascular 
events). For AF, incidence rates were also calculated per age group and for men and women separately. For the outcomes, incidence rates were calculated separately for participants with screen-detected AF, participants with clinically detected AF and participants without AF. For the outcomes, separate incidence rates were also calculated for events occurring before $\mathrm{AF}$ and events occurring after $\mathrm{AF}$.

Next, we used Cox regression models to assess the association of screen-detected $\mathrm{AF}$ and clinically detected $\mathrm{AF}$ with all-cause mortality, incident $\mathrm{HF}$ and vascular events. In order to account for the fact that events could occur prior to incident AF, screen-detected $\mathrm{AF}$ and clinically detected $\mathrm{AF}$ were entered into the Cox regression models as timevarying covariates. Thus, participants who developed incident $\mathrm{AF}$ were counted as participants without $\mathrm{AF}$ prior to the moment of $\mathrm{AF}$ diagnosis (non-exposed time) and were counted as participants with $\mathrm{AF}$ from the moment of AF diagnosis onwards (exposed time). For each outcome, we built three Cox regression models: (1) an unadjusted model including screen-detected $\mathrm{AF}$ and hospital-AF only, (2) model 1 additionally adjusted for age and sex and (3) model 2 additionally adjusted for relevant clinical characteristics (hypertension, diabetes, stroke, MI, peripheral artery disease, prevalent HF and natriuretic peptides). ${ }^{18}$ In order to assess whether screen-detected $\mathrm{AF}$ was significantly more or significantly less associated with outcomes compared with clinically detected AF, we calculated $p$ values for the difference in HRs between both AF types. The Cox proportional hazards assumption was assessed graphically using Kaplan-Meier curves for each outcome, ${ }^{21}$ which revealed no violations of the proportional hazards assumption. For analyses with incident HF as the outcome, we excluded participants with prevalent HF $(n=18)$. Given the design of PREVEND with overselection of individuals with microalbuminuria, all
Cox regression analyses were adjusted for baseline UAC. Analyses were performed using SPSS V.25. A p value $<0.05$ was considered statistically significant.

\section{RESULTS}

\section{AF incidence and participant characteristics}

The included PREVEND participants had a mean age of $49 \pm 13$ years, and $50 \%$ were women. During a mean follow-up duration of $9.8 \pm 2.3$ years, 265 individuals $(3.2 \%)$ developed incident AF. Of all incident AF cases, 60 (23\%) were screen-detected and 205 (77\%) were clinically detected. In men, $26 \%$ of incident $\mathrm{AF}$ cases were screen-detected, compared with $15 \%$ for women $(\mathrm{p}=0.051)$. AF incidence rates were higher for older compared with younger participants, and for men compared with women (figure 1).

Compared with those with clinically detected AF, participants with screen-detected AF less often had peripheral artery disease or elevated natriuretic peptides (table 1). Otherwise, there were no significant baseline differences between participants with screen-detected and clinically detected AF.

\section{Outcomes}

During the follow-up period, 605 (7.3\%) participants died, 262 (3.2\%) developed incident HF and 806 (9.8\%) had a vascular event. In the screen-detected AF group, $9(15.0 \%)$ participants died, $11(18.3 \%)$ developed incident $\mathrm{HF}$ and $12(20.0 \%)$ had at least one vascular event. In the clinically detected AF group, 64 (32.2\%) participants died, 71 (34.6\%) developed incident HF and 100 (48.8\%) had a vascular event.

In participants with clinically detected AF, the majority of incident HF cases and vascular events occurred prior to $\mathrm{AF}$ diagnosis, which was not the case in those with

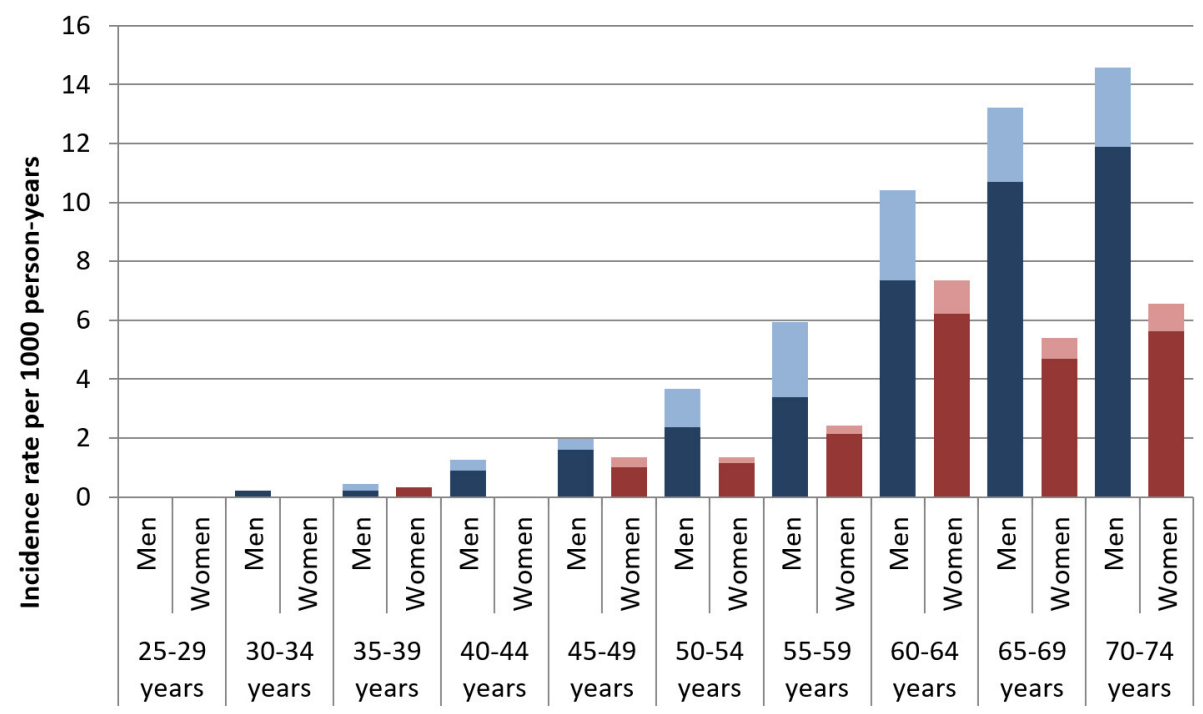

- Clinically detected AF $\square$ Screen-detected AF

Figure 1 Incidence rates per 1000 person-years of screen-detected and clinically detected atrial fibrillation per age category in men and women. AF, atrial fibrillation. 


\begin{tabular}{|c|c|c|c|c|}
\hline Clinical characteristics & $\begin{array}{l}\text { Total study population } \\
(\mathrm{n}=8265)\end{array}$ & $\begin{array}{l}\text { Screen-detected AF } \\
(n=60)\end{array}$ & $\begin{array}{l}\text { Clinically detected AF } \\
(n=205)\end{array}$ & $P$ value \\
\hline Male sex & $4120(50 \%)$ & $48(80 \%)$ & $137(67 \%)$ & 0.051 \\
\hline Age & $49 \pm 13$ & $60 \pm 8$ & $62 \pm 9$ & 0.163 \\
\hline $\mathrm{BMI}\left(\mathrm{kg} / \mathrm{m}^{2}\right)$ & $26 \pm 4$ & $28 \pm 5$ & $28 \pm 4$ & 0.247 \\
\hline Hypertension & $2613(32 \%)$ & $41(68 \%)$ & $138(67 \%)$ & 0.882 \\
\hline Diabetes & $310(4 \%)$ & $4(7 \%)$ & $19(9 \%)$ & 0.562 \\
\hline History of MI & $251(3 \%)$ & $5(8 \%)$ & $36(18 \%)$ & 0.084 \\
\hline History of stroke & $81(1 \%)$ & $3(5 \%)$ & $6(3 \%)$ & 0.428 \\
\hline History of PAD & $291(4 \%)$ & $2(3 \%)$ & $26(13 \%)$ & 0.032 \\
\hline Previously diagnosed HF & $18(0.2 \%)$ & $1(2 \%)$ & $5(2 \%)$ & 1.000 \\
\hline NT-proBNP > 125 ng/L & $926(11 \%)$ & $18(30 \%)$ & $93(45 \%)$ & 0.048 \\
\hline eGFR $<60 \mathrm{~mL} / \mathrm{min} / 1,73 \mathrm{~m}^{2}$ & $466(6 \%)$ & $3(5 \%)$ & $18(9 \%)$ & 0.425 \\
\hline $\mathrm{UAC} \geq 10 \mathrm{mg} / \mathrm{L}$ & $5759(70 \%)$ & $45(75 \%)$ & $159(78 \%)$ & 0.679 \\
\hline $\mathrm{CHA}_{2} \mathrm{DS}_{2}$-VASc score & $1.1 \pm 1.0$ & $1.5 \pm 1.2$ & $2.0 \pm 1.1$ & 0.192 \\
\hline
\end{tabular}

Data presented as mean \pm SD or count (\%). $\mathrm{P}$ values represent the difference between participants with screen-detected $A F$ and clinically detected $A F$.

AF, atrial fibrillation; BMI, body mass index ; eGFR, estimated glomerular filtration rate; HF, heart failure; MI, myocardial infarction; NTproBNP, N-terminal pro-brain natriuretic peptide; PAD, peripheral artery disease; UAC, urinary albumin concentration.

screen-detected AF (figure 2). In both groups, coronary events (ie, events related to myocardial ischaemia) made up the majority of vascular events, and the incidence of stroke was relatively low (figure 3). Incidence rates for outcomes occurring after AF diagnosis were generally higher in participants with clinically detected $\mathrm{AF}$ than in participants with screen-detected AF, although CIs overlapped (figure 3).

In the unadjusted Cox regression analyses, both screendetected $\mathrm{AF}$ and clinically detected AF were significantly associated with all-cause mortality, incident HF and vascular events. The corresponding HRs were not

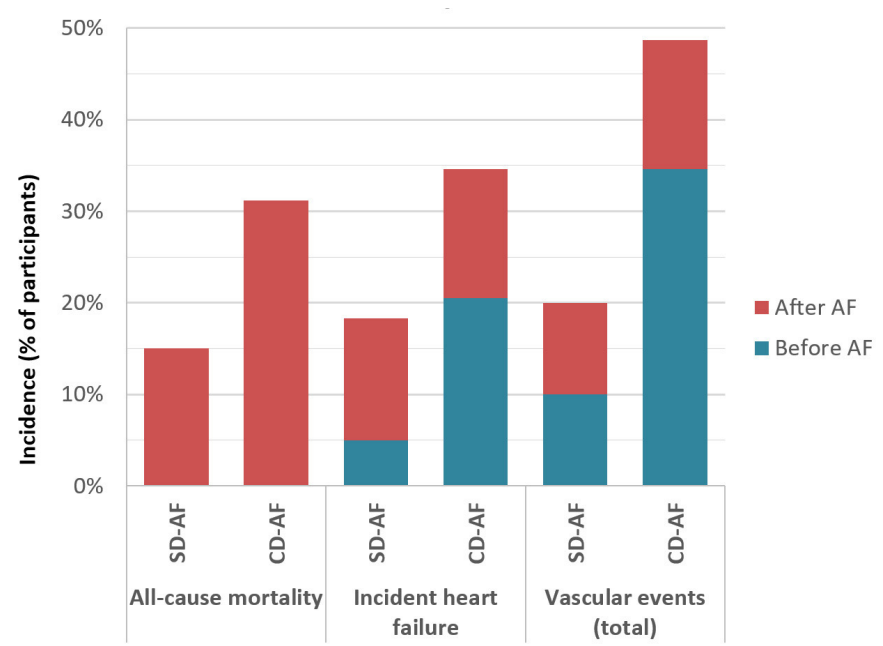

Figure 2 Incidence of outcomes before and after diagnosis of atrial fibrillation. AF, atrial fibrillation; CD-AF, clinically detected atrial fibrillation; SD-AF, screen-detected atrial fibrillation. significantly different between screen-detected $\mathrm{AF}$ and clinically detected AF (table 2).

After adjustment for age and sex, screen-detected AF was still significantly associated with all-cause mortality and incident HF, but not with vascular events. Clinically detected AF remained significantly associated with all three outcomes after adjustment for age and sex. There were no significant differences between the HRs of screen-detected $\mathrm{AF}$ and clinically detected $\mathrm{AF}$ (table 2).

After multivariable adjustment for risk factors and comorbidities, screen-detected AF remained significantly associated with all-cause mortality (HR 2.21, 95\% CI 1.09 to 4.47 ) and incident HF (HR 4.90, 95\% CI 2.28 to 10.57), but not with vascular events (HR 1.12, 95\% CI 0.46 to 2.71). Clinically detected AF remained significantly associated with all-cause mortality (HR 2.95, 95\% CI 2.18 to 4.00), incident HF (HR 3.98, 95\% CI 2.49 to 6.34 ) and vascular events (HR 1.92, 95\% CI 1.21 to 3.06). The multivariable adjusted HRs were not statistically different between screen-detected $\mathrm{AF}$ and clinically detected $\mathrm{AF}$ for all-cause mortality ( $p$ for difference $=0.447$ ), incident HF ( $p$ for difference $=0.635$ ) or vascular events ( $p$ for difference $=0.283)$.

\section{DISCUSSION}

In this study, we demonstrated that screen-detected AF is associated with adverse outcomes, particularly allcause mortality and incident $\mathrm{HF}$, in participants from the population-based PREVEND study. We did not find significant differences between screen-detected $\mathrm{AF}$ and clinically detected $\mathrm{AF}$ in terms of their association with mortality, incident $\mathrm{HF}$ or vascular events. 


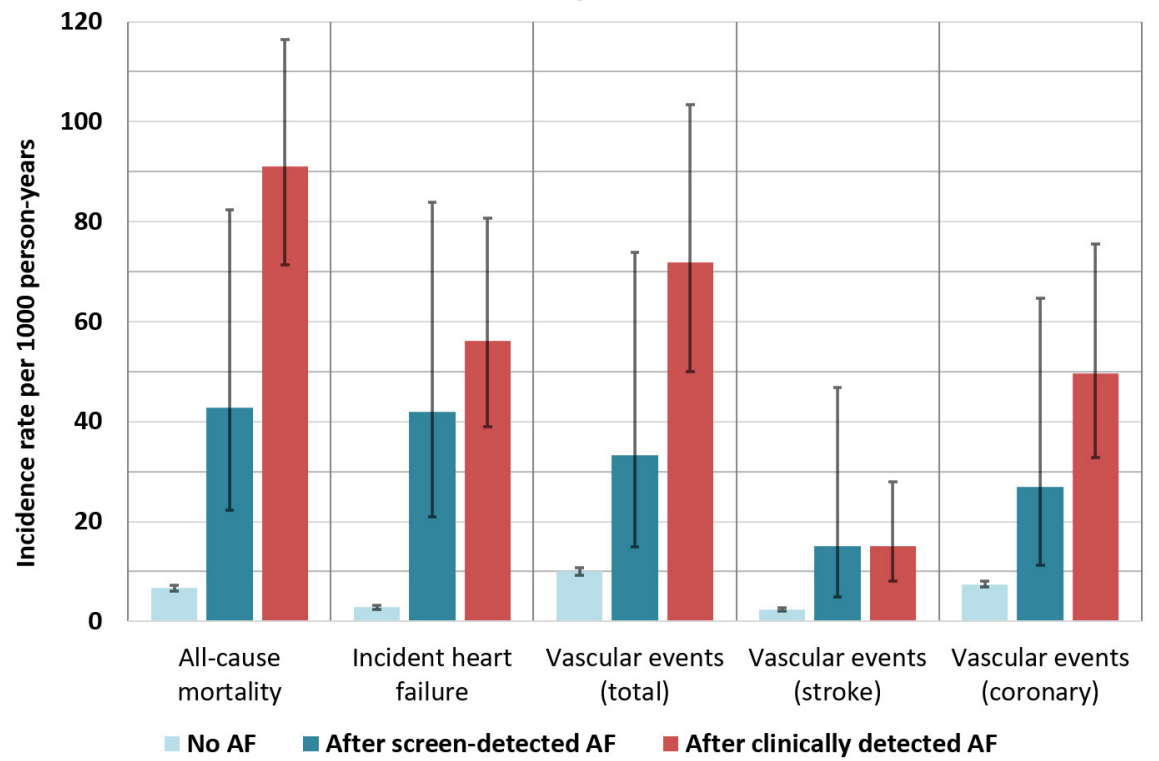

Figure 3 Outcome incidence rates per 1000 person-years after diagnosis of atrial fibrillation. AF, atrial fibrillation.

\section{Outcomes in screen-detected AF}

In recent years, a wide range of screening methods have been shown to improve early detection of AF. ${ }^{11}$ While it is generally assumed that screen-detected AF is associated with adverse outcomes, only few studies have actually assessed the risk of adverse outcomes in screen-detected

\begin{tabular}{|c|c|c|c|c|c|}
\hline Outcomes & $\begin{array}{l}\text { Time-varying } \\
\text { covariate }\end{array}$ & $\begin{array}{l}\text { HR } \\
(95 \% \mathrm{Cl})\end{array}$ & $P$ value & $\begin{array}{l}\text { HR(SD-AF) / HR(CD-AF) } \\
(95 \% \mathrm{CI})\end{array}$ & P for difference \\
\hline \multicolumn{6}{|l|}{ Model 1: unadjusted } \\
\hline \multirow[t]{2}{*}{ All-cause mortality } & Screen-detected AF & 5.66 (2.92 to 10.98$)$ & $<0.001$ & 0.52 (0.26 to 1.06$)$ & 0.070 \\
\hline & Clinically detected AF & 10.83 (8.23 to 14.26$)$ & $<0.001$ & & \\
\hline \multirow[t]{2}{*}{ Incident heart failure } & Screen-detected AF & 12.10 (5.93 to 24.70$)$ & $<0.001$ & 1.02 (0.45 to 2.29$)$ & 0.970 \\
\hline & Clinically detected AF & 11.92 (7.63 to 18.61$)$ & $<0.001$ & & \\
\hline \multirow[t]{2}{*}{ Vascular events } & Screen-detected AF & 3.27 (1.46 to 7.33 ) & 0.004 & 0.73 (0.29 to 1.83$)$ & 0.505 \\
\hline & Clinically detected AF & 4.47 (2.84 to 7.05$)$ & $<0.001$ & & \\
\hline \multicolumn{6}{|c|}{ Model 2: adjusted for age and sex } \\
\hline \multirow[t]{2}{*}{ All-cause mortality } & Screen-detected AF & 2.24 (1.16 to 4.36$)$ & 0.017 & 0.58 (0.29 to 1.16$)$ & 0.122 \\
\hline & Clinically detected AF & 3.90 (2.96 to 5.13$)$ & $<0.001$ & & \\
\hline \multirow[t]{2}{*}{ Incident heart failure } & Screen-detected AF & 5.16 (2.52 to 10.58$)$ & $<0.001$ & 1.12 (0.50 to 2.53$)$ & 0.780 \\
\hline & Clinically detected AF & 4.60 (2.93 to 7.21$)$ & $<0.001$ & & \\
\hline \multirow[t]{2}{*}{ Vascular events } & Screen-detected AF & 1.24 (0.55 to 2.79$)$ & 0.598 & 0.64 (0.25 to 1.58$)$ & 0.330 \\
\hline & Clinically detected AF & 1.96 (1.25 to 3.07$)$ & 0.003 & & \\
\hline \multicolumn{6}{|c|}{ Model 3: adjusted for age, sex, prevalent heart failure, hypertension, diabetes, stroke, myocardial infarction, peripheral artery disease, and NT-proBNP } \\
\hline \multirow[t]{2}{*}{ All-cause mortality } & Screen-detected AF & $2.21(1.09$ to 4.47$)$ & 0.028 & 0.75 (0.35 to 1.58$)$ & 0.447 \\
\hline & Clinically detected AF & $2.95(2.18$ to 4.00$)$ & $<0.001$ & & \\
\hline \multirow[t]{2}{*}{ Incident heart failure } & Screen-detected AF & $4.90(2.28$ to 10.57$)$ & $<0.001$ & 1.23 (0.52 to 2.92) & 0.635 \\
\hline & Clinically detected AF & $3.98(2.49$ to 6.34$)$ & $<0.001$ & & \\
\hline \multirow[t]{2}{*}{ Vascular events } & Screen-detected AF & 1.12 (0.46 to 2.71$)$ & 0.802 & 0.58 (0.22 to 1.56$)$ & 0.283 \\
\hline & Clinically detected AF & 1.92 (1.21 to 3.06$)$ & 0.006 & & \\
\hline
\end{tabular}

$\mathrm{P}$ values denote the significance of the time-varying covariates (screen-detected AF or clinically detected AF) in terms of their association with each clinical outcome (all-cause mortality, incident heart failure or vascular events). The $P$ values for difference represent the difference in HRs between screen-detected AF and clinically detected AF.

AF, atrial fibrillation; CD-AF, clinically detected AF; NT-proBNP, N-terminal pro-brain natriuretic peptide; SD-AF, screen-detected AF. 
$\mathrm{AF}$, or studied how this risk compares to the risk in clinically detected AF.

In a recent pharmacy-based opportunistic screening study, which used single time point hand-held ECGs, the risk of all-cause mortality and CV hospitalisation was significantly higher in participants with screen-detected $\mathrm{AF}$ compared with those without $\mathrm{AF}$, and there were no significant differences in risk between screen-detected $\mathrm{AF}$ and known prevalent $\mathrm{AF}^{22}$ Although we compared screen-detected AF with clinically detected incident $\mathrm{AF}$ (rather than prevalent $\mathrm{AF}$ ), our findings seem to be in line with the results of this pharmacy-based screening study.

Other studies have mostly focused on outcomes in two populations that are somewhat different, yet related to our population with screen-detected AF: (1) patients with subclinical AF (SCAF) and atrial high-rate episodes (AHRE) as detected by cardiac implantable electronic devices (CIEDs) and (2) patients with asymptomatic (silent) AF.

Studies in patients with CIEDs, such as the Asymptomatic Atrial Fibrillation and Stroke Evaluation in Pacemaker Patients and the Atrial Fibrillation Reduction Atrial Pacing Trial (ASSERT), have demonstrated that SCAF/AHRE is associated with vascular events, HF hospitalisation and mortality. ${ }^{23-26}$ Our results seem largely in line with these studies. However, comparisons between SCAF/AHRE and screen-detected AF should be made with caution, since patients with CIEDs may be different from the general AF population. ${ }^{27}$

Studies in asymptomatic AF are also relevant to the topic of screen-detected AF, since individuals with screendetected AF are frequently asymptomatic. ${ }^{27-30}$ In the EurObservational Research Programme - Atrial Fibrillation (EORP-AF) registry, patients with asymptomatic AF had a substantially higher risk of mortality compared with their symptomatic counterparts, although it has to be noted that asymptomatic patients were less often treated with oral anticoagulants (OACs) ${ }^{31}$ By contrast, in patients with persistent AF from the RAte Control versus Electrical cardioversion for persistent atrial fibrillation (RACE) study, asymptomatic AF was associated with a lower risk of $\mathrm{CV}$ morbidity and mortality than symptomatic AF, although the difference may in part have been caused by the lower prevalence of HF in the asymptomatic group. ${ }^{32}$ Our findings seem more in line with recent results from the Global Anticoagulant Registry in the Field - Atrial Fibrillation (GARFIELD-AF) study, which found no difference in outcomes between asymptomatic and symptomatic patients with at least one stroke risk factor. ${ }^{33}$ Importantly, studies in asymptomatic $\mathrm{AF}$ have some limitations when applied to the setting of $\mathrm{AF}$ screening. First, many of these studies were performed in selected (mostly hospital-based) populations, which may be different from the general population targeted with screening. Furthermore, not all individuals with screen-detected AF are asymptomatic: in the populationbased Lifelines study, which used single time point ECG screening similar to PREVEND, palpitations were in fact a major independent predictor of previously undiagnosed $\mathrm{AF}^{34}$

After multivariable adjustment, screen-detected AF remained significantly associated with all-cause mortality and incident HF, but not with vascular events. This might be due to the small number of vascular events, particularly stroke, that occurred in participants with screendetected AF. The low incidence of stroke seems consistent with previous AF screening trials ${ }^{22} 27$ and could possibly be explained by initiation OACs after diagnosis of screendetected AF. Based on our data, we cannot draw definitive conclusions about the risk of stroke and other vascular events in (untreated) screen-detected AF. However, recently published results from the STROKESTOP II trial indicate that $\mathrm{AF}$ screening and subsequent treatment initiation may lead to a modest, yet significant reduction of stroke incidence in the general population. ${ }^{35}$

Overall, in our study, the association of screen-detected $\mathrm{AF}$ with incident $\mathrm{HF}$ was stronger than its association with mortality and vascular events. This highlights that future studies should focus not only on preventing stroke and mortality but also on preventing $\mathrm{HF}$ in patients with screen-detected $\mathrm{AF}^{24}$

\section{Clinical characteristics of individuals with screen-detected AF and clinically detected AF}

Another aim of our study was to compare clinical characteristics between individuals with screen-detected and clinically detected AF. We found that both groups had largely similar baseline characteristics, with only a few differences. Participants with clinically detected AF had higher natriuretic peptide levels at baseline, which may indicate that they were more likely to have previously unrecognised or subclinical $\mathrm{HF}$ at baseline, and that $\mathrm{AF}$ may have been detected during hospital visits for HF. Indeed, in participants with clinically detected $\mathrm{AF}$, the majority of incident $\mathrm{HF}$ cases were diagnosed prior to $\mathrm{AF}$. By contrast, HF was mostly diagnosed after AF in participants with screen-detected AF. Furthermore, the higher proportion of women in the clinically detected AF group seems in line with previous studies in symptomatic versus asymptomatic $\mathrm{AF}$, which have consistently demonstrated that patients with symptomatic AF are more likely to be women compared with asymptomatic individuals. ${ }^{31-33}$

\section{Strengths and limitations}

Our study was performed in a large population-based cohort with almost 10 years of follow-up and solid validation of incident $\mathrm{AF}$ and endpoints. Due to the linkage with hospital registries, we were able to provide a reliable and direct comparison between screen-detected and clinically detected AF.

Limitations include the lack of data about type of AF (paroxysmal, persistent or permanent) and symptoms. This information should be taken into account in future studies, as it may help explain why AF remains unrecognised in some but not in others. During the three-yearly 
PREVEND visits, only single time point ECGs were made, and, therefore, cases of paroxysmal AF may have been missed. Although the use of ICD-9-based discharge codes from the Dutch National Medical Registry has been shown to be highly reliable ${ }^{18}{ }^{36}$ we cannot fully rule out that some vascular outcomes were missed or misclassified. Data on the primary reason for the hospitalisations or outpatient visits during which $\mathrm{AF}$ was diagnosed were not available. Furthermore, data regarding medication use during follow-up were unavailable: since OACs may have been initiated in a large proportion of individuals with incident $\mathrm{AF}$, the incidence of vascular events in our study was likely an underestimation. Since we used data up to 2008, results might be different in the present-day setting due to advances in the treatment of AF in recent years. Finally, the PREVEND cohort is predominantly Caucasian and enriched for microalbuminuria, which may limit generalisability.

\section{CONCLUSIONS}

This study demonstrates that individuals with screendetected AF are at increased risk of adverse outcomes, particularly all-cause mortality and incident HF. We did not find significant differences in risk between screendetected and clinically detected AF. However, randomised trials are needed to assess if outcomes can be improved by treating individuals with screen-detected AF similarly to patients with clinically detected AF. Our results highlight that AF screening trials should focus not only on the prevention of stroke and mortality but also on the prevention of $\mathrm{HF}$ in individuals with screen-detected $\mathrm{AF}$.

\section{Author affiliations}

${ }^{1}$ Department of Cardiology, University Medical Center Groningen, University of Groningen, Groningen, The Netherlands

${ }^{2}$ Department of Internal Medicine, Nephrology Division, University Medical Center Groningen, University of Groningen, Groningen, The Netherlands

Contributors BG, NS, RTG, SJLB, RAdB and MR contributed to the conception, design, and acquisition of the work. VWZ, BG, NS, ICvG, RAdB and MR contributed to the data analysis and interpretation. VWZ and MR drafted the manuscript and are responsible for the overall content of the work as guarantors. All authors contributed to the critical revision of the manuscript, gave final approval of the version to be published and agree to be accountable for all aspects of the work.

Funding This work was supported by the Dutch Heart Foundation [CVON RED-CVD, grant 2017-11]. The PREVEND study was supported by the Dutch Kidney Foundation [grant E0.13].

Competing interests For the current manuscript, the authors declare no potential conflicts of interest. Outside of the submitted work, the authors disclosed the following financial support: RAdB. reports grants from the Dutch Heart Foundation (CVON SHE-PREDICTS-HF, grant 2017-21; CVON RED-CVD, grant 2017-11; CVON PREDICT2, grant 2018-30; and CVON DOUBLE DOSE, grant 2020B005), leDucq Foundation (Cure PhosphoLambaN induced Cardiomyopathy (Cure-PLaN)), and the European Research Council (SECRETE-HF, ERC CoG 818715). The UMCG, which employs RAdB., has received research grants and/or fees from AstraZeneca, Abbott, Boehringer Ingelheim, Cardior Pharmaceuticals Gmbh, Ionis Pharmaceuticals, Inc., Novo Nordisk, and Roche. RAdB. has received speaker fees from Abbott, AstraZeneca, Bayer, Novartis, and Roche. MR reports grants from the Dutch Heart Foundation (CVON RACE V, grant 2014-09; CVON RED-CVD, grant 2017-11; CVONAl, grant 2018B017; DECISION, grant 2018B024). The UMCG, which employs MR, has received grants from SJM/Abbott (VIP-HF study) and Medtronic (Cryoballoon AF registry/biobank study).
Patient consent for publication Not applicable.

Ethics approval This study involves human participants and was approved by Medical Ethical Committee of the University Medical Center Groningen. Participants gave informed consent to participate in the study before taking part.

Provenance and peer review Not commissioned; externally peer reviewed.

Data availability statement Data are available upon reasonable request. For more information, please visit: https://groningendatacatalogus.nl/menu/main/ dataexplorer/details/umcg_collections/17.

Open access This is an open access article distributed in accordance with the Creative Commons Attribution Non Commercial (CC BY-NC 4.0) license, which permits others to distribute, remix, adapt, build upon this work non-commercially, and license their derivative works on different terms, provided the original work is properly cited, appropriate credit is given, any changes made indicated, and the use is non-commercial. See: http://creativecommons.org/licenses/by-nc/4.0/.

\section{ORCID iDs}

Victor W Zwartkruis http://orcid.org/0000-0003-0115-1313

Navin Suthahar http://orcid.org/0000-0002-7781-2897

Stephan J L Bakker http://orcid.org/0000-0003-3356-6791

Rudolf A de Boer http://orcid.org/0000-0002-4775-9140

Michiel Rienstra http://orcid.org/0000-0002-2581-070X

\section{REFERENCES}

1 Hindricks G, Potpara T, Dagres N. ESC Guidelines for the diagnosis and management of atrial fibrillation developed in collaboration with the European Association for Cardio-Thoracic Surgery (EACTS). Eur Heart J 2020;2021:373-498.

2 Börschel CS, Schnabel RB. The imminent epidemic of atrial fibrillation and its concomitant diseases - Myocardial infarction and heart failure - A cause for concern. Int $J$ Cardiol 2019;287:162-73.

3 Ruddox V, Sandven I, Munkhaugen J, et al. Atrial fibrillation and the risk for myocardial infarction, all-cause mortality and heart failure: a systematic review and meta-analysis. Eur J Prev Cardiol 2017;24:1555-66.

4 Kriithe BP, Kunst A, Benjamin EJ, et al. Projections on the number of individuals with atrial fibrillation in the European Union, from 2000 to 2060. Eur Heart J 2013;34:2746-51.

5 Freedman B, Camm J, Calkins H, et al. Screening for atrial fibrillation. Circulation 2017;135:1851-67.

6 Hart RG, Pearce LA, Aguilar MI. Meta-analysis: antithrombotic therapy to prevent stroke in patients who have nonvalvular atrial fibrillation. Ann Intern Med 2007;146:857-67.

7 Ruff CT, Giugliano RP, Braunwald E, et al. Comparison of the efficacy and safety of new oral anticoagulants with warfarin in patients with atrial fibrillation: a meta-analysis of randomised trials. Lancet 2014;383:955-62.

8 Kirchhof P, Camm AJ, Goette A, et al. Early Rhythm-Control Therapy in Patients with Atrial Fibrillation. N Engl J Med 2020;383:1305-16.

9 Engdahl J, Svennberg E, Friberg L, et al. Stepwise mass screening for atrial fibrillation using $\mathrm{N}$-terminal pro B-type natriuretic peptide: the STROKESTOP II study design. Europace 2017;19:297-302.

10 Lowres N, Olivier J, Chao T-F, et al. Estimated stroke risk, yield, and number needed to screen for atrial fibrillation detected through single time screening: a multicountry patient-level meta-analysis of 141,220 screened individuals. PLoS Med 2019;16:1-19.

11 Petryszyn P, Niewinski P, Staniak A, et al. Effectiveness of screening for atrial fibrillation and its determinants. A meta-analysis. PLoS One 2019;14:1-17.

12 Lopes RD, Alings M, Connolly SJ, et al. Rationale and design of the Apixaban for the Reduction of Thrombo-Embolism in Patients With Device-Detected Sub-Clinical Atrial Fibrillation (ARTESiA) trial. Am Heart J 2017;189:137-45.

13 Kirchhof P, Blank BF, Calvert M, et al. Probing oral anticoagulation in patients with atrial high rate episodes: rationale and design of the Non-vitamin $\mathrm{K}$ antagonist Oral anticoagulants in patients with Atrial High rate episodes (NOAH-AFNET 6) trial. Am Heart $J$ 2017;190:12-18.

14 University of Cambridge. The SAFER trial - Screening for Atrial Fibrillation with ECG to Reduce stroke. Available: https://www.safer. phpc.cam.ac.uk/ [Accessed 14 Jul 2021].

15 Benjamin EJ, Go AS, Desvigne-Nickens P, et al. Research priorities in atrial fibrillation screening: a report from a National Heart, Lung, and Blood Institute virtual workshop. Circulation 2021;143:372-88. 
16 Hillege HL, Fidler V, Diercks GFH, et al. Urinary albumin excretion predicts cardiovascular and noncardiovascular mortality in general population. Circulation 2002;106:1777-82.

17 Brouwers FP, de Boer RA, van der Harst P, et al. Incidence and epidemiology of new onset heart failure with preserved vs. reduced ejection fraction in a community-based cohort: 11-year follow-up of PREVEND. Eur Heart J 2013;34:1424-31.

18 Vermond RA, Geelhoed B, Verweij N, et al. Incidence of atrial fibrillation and relationship with cardiovascular events, heart failure, and mortality: a community-based study from the Netherlands. J Am Coll Cardiol 2015;66:1000-7.

19 Suthahar N, Meems LMG, van Veldhuisen DJ, et al. HighSensitivity troponin-T and cardiovascular outcomes in the community: differences between women and men. Mayo Clin Proc 2020;95:1158-68.

20 CBS - Statistics Netherlands [Data specifically requested]. Available: https://www.cbs.nl/en-gb [Accessed 14 Jul 2021].

21 Bellera CA, MacGrogan G, Debled M, et al. Variables with timevarying effects and the Cox model: some statistical concepts illustrated with a prognostic factor study in breast cancer. BMC Med Res Methodol 2010;10:20.

22 Zink MD, Mischke KG, Keszei AP, et al. Screen-detected atrial fibrillation predicts mortality in elderly subjects. Europace 2021;23:29-38.

23 Van Gelder IC, Healey JS, Crijns HJGM, et al. Duration of devicedetected subclinical atrial fibrillation and occurrence of stroke in ASSERT. Eur Heart J 2017;38:1339-44.

24 Wong JA, Conen D, Van Gelder IC, et al. Progression of DeviceDetected Subclinical Atrial Fibrillation and the Risk of Heart Failure. J Am Coll Cardiol 2018;71:2603-11.

25 Gonzalez M, Keating RJ, Markowitz SM, et al. Newly detected atrial high rate episodes predict long-term mortality outcomes in patients with permanent pacemakers. Heart Rhythm 2014;11:2214-21.

26 Mahajan R, Perera T, Elliott AD, et al. Subclinical device-detected atrial fibrillation and stroke risk: a systematic review and metaanalysis. Eur Heart J 2018;39:1407-15.
27 Engdahl J, Holmén A, Rosenqvist M, et al. A prospective 5-year follow-up after population-based systematic screening for atrial fibrillation. Europace 2018;20:f306-11.

28 Chan N-Y, Choy C-C. Screening for atrial fibrillation in 13122 Hong Kong citizens with smartphone electrocardiogram. Heart 2017;103:24-31.

29 Steinhubl SR, Waalen J, Edwards AM, et al. Effect of a homebased wearable continuous ECG monitoring patch on detection of undiagnosed atrial fibrillation: the mSToPS randomized clinical trial. JAMA 2018;320:146-55.

30 Gladstone DJ, Wachter R, Schmalstieg-Bahr K, et al. Screening for atrial fibrillation in the older population: a randomized clinical trial. JAMA Cardiol 2021;6:558-67.

31 Boriani G, Laroche C, Diemberger I, et al. Asymptomatic atrial fibrillation: clinical correlates, management, and outcomes in the EORP-AF pilot general registry. Am J Med 2015;128:509-18.

32 Rienstra M, Vermond RA, Crijns HJGM, et al. Asymptomatic persistent atrial fibrillation and outcome: results of the RACE study. Heart Rhythm 2014;11:939-45.

33 Gibbs H, Freedman B, Rosenqvist M, et al. Clinical outcomes in asymptomatic and symptomatic atrial fibrillation presentations in GARFIELD-AF: implications for AF screening. Am J Med 2021;134:893-901.

34 Zwartkruis VW, Groenewegen A, Rutten FH, et al. Proactive screening for symptoms: a simple method to improve early detection of unrecognized cardiovascular disease in primary care. Results from the Lifelines cohort study. Prev Med 2020;138:106143.

35 Svennberg E, Friberg L, Frykman V, et al. Clinical outcomes in systematic screening for atrial fibrillation (STROKESTOP): a multicentre, parallel group, unmasked, randomised controlled trial. Lancet 2021;398:1498-506.

36 Stricker BHC, Herings RMC. Pleidooi voor het behoud van de Landelijke Medische Registratie voor betrouwbare informatie over de volksgezondheid en de gezondheidszorg. Ned Tijdschr Geneeskd 2006;150:1916-7. 